\title{
Análise da Revisão Cochrane: Anti-fator de Crescimento \\ Vascular Endotelial na Prevenção da Hemorragia \\ Vítrea Pós-Operatória após Vitrectomia por Retinopatia \\ Diabética Proliferativa. Cochrane Database Syst Rev. \\ 2015;8:CD008214.
}

\author{
Analysis of the Cochrane Review: Anti-vascular \\ Endothelial Growth Factor for Prevention of Postoperative \\ Vitreous Cavity Hemorrhage after Vitrectomy for \\ Proliferative Diabetic Retinopathy. Cochrane Database \\ Syst Rev. 2015;8:CD008214.
}

David Cordeiro SOUSA ${ }^{1,2}$, Inês LEAL ${ }^{1,2}$, João Costa ${ }^{3,4}$, António VAZ-CARNEIRO $\square^{3,4}$

Acta Med Port 2017 Jul-Aug;30(7-8):513-516 - https://doi.org/10.20344/amp.9437

\begin{abstract}
RESUMO
A hemorragia vítrea pós-operatória é uma complicação da cirurgia de vitrectomia por retinopatia diabética proliferativa, que atrasa a melhoria da acuidade visual e dificulta a visualização do fundo ocular e o seguimento da doença. Quando injetados na cavidade vítrea, os fármacos anti-factor de crescimento do endotélio vascular, como o bevacizumab, diminuem a proliferação vascular e o seu uso tem sido proposto na redução da incidência de hemorragia vítrea pós-operatória. Os autores desta revisão sistemática Cochrane reviram os ensaios clínicos aleatorizados sobre o uso pré- ou intraoperatório de anti-factor de crescimento do endotélio vascular na redução da incidência de hemorragia vítrea pós-operatória após vitrectomia em doentes com retinopatia diabética proliferativa. Dos resultados, destaca-se o efeito benéfico da utilização de bevacizumab na diminuição da hemorragia vítrea pós-operatória precoce (i.e. às quatro semanas), com um bom perfil de segurança. Neste trabalho, sumarizam-se e discutem-se os principais achados e implicações práticas desta revisão sistemática Cochrane.

Palavras-chave: Bevacizumab; Factores de Crescimento do Endotélio Vascular/ antagonistas \& inibidores; Hemorragia PósOperatória/prevenção e controlo; Hemorragia Vítrea/prevenção e controlo; Revisão Sistemática; Retinopatia Diabética; Vitrectomia/ efeitos adversos
\end{abstract}

\section{ABSTRACT}

Postoperative vitreous hemorrhage is a complication following vitrectomy for proliferative diabetic retinopathy, delaying visual recovery and making fundus examination and disease follow-up more difficult. Anti-vascular endothelial growth factor drugs such as bevacizumab, when injected in the vitreous cavity, reduce vascular proliferation and their use has been proposed to reduce the incidence of postoperative vitreous hemorrhage. The authors of this Cochrane systematic review evaluated all randomized controlled trials on the pre- or intraoperative use of anti-vascular endothelial growth factor to reduce postoperative vitreous hemorrhage occurrence after vitrectomy in patients with proliferative diabetic retinopathy. The results suggested that the use of intravitreal bevacizumab was effective in reducing early postoperative vitreous hemorrhage (i.e. at four weeks) occurrence, with a good safety profile. This work aims to summarize and discuss the findings and clinical implications of this Cochrane systematic review.

Keywords: Bevacizumab; Diabetic Retinopathy; Postoperative Hemorrhage/prevention \& control; Randomized Controlled Trials as Topic; Vascular Endothelial Growth Factors/antagonists \& inhibitors; Vitrectomy/adverse effects; Vitreous Hemorrhage/prevention \& control

\section{QUESTÃO CLÍNICA}

A utilização de fármacos anti-factor de crescimento do endotélio vascular (anti-VEGF) está associada a uma diminuição da ocorrência de hemorragia vítrea pós-operatória (HVPO) após vitrectomia por retinopatia diabética proliferativa (RDP)?

\section{OBJECTIVOS}

Avaliar e analisar a evidência atual relativamente à efi- cácia da utilização pré- ou intraoperatória de anti-VEGF na redução da incidência de HVPO após vitrectomia por RDP.

\section{METODOLOGIA}

Esta revisão sistemática (RS) é uma atualização do trabalho sobre o mesmo tema, de maio de $2011 .{ }^{1}$ Foram pesquisadas as seguintes bases de dados: CENTRAL, Ovid MEDLINE, EMBASE, PubMed, Latin American and Caribbean Health Sciences, the ISRCTN meta Register of

1. Serviço de Oftalmologia. Hospital de Santa Maria. Centro Hospitalar Lisboa Norte. Lisboa. Portugal.

2. Centro de Estudos das Ciências da Visão. Faculdade de Medicina. Universidade de Lisboa. Lisboa. Portugal.

3. Centro de Estudos de Medicina Baseada na Evidência. Faculdade de Medicina. Universidade de Lisboa. Lisboa. Portugal.

4. Cochrane Portugal. Lisboa. Portugal.

$\square$ Autor correspondente: António Vaz Carneiro. avc@medicina.ulisboa.pt

Recebido: 14 de junho de 2017 - Aceite: 14 de junho de 2017 | Copyright @ Ordem dos Médicos 2017 
Controlled Trials, ClinicalTrials.gov, e WHO International Clinical Trials Registry Platform. Não foi utilizada nenhuma restrição de idioma ou data, tendo a última pesquisa sido realizada a 26 de maio de 2015.

Incluíram-se todos os ensaios clínicos aleatorizados e controlados (EAC) e quasi-EAC, que estudaram a influência da utilização intravítrea de fármacos anti-VEGF na incidência de HVPO em doentes que realizaram vitrectomia por RDP, com um mínimo de 6 meses de seguimento pós-operatório.

As principais medidas de eficácia foram: i) a incidência de HVPO precoce (até às quatro semanas pós-operatórias) e tardia (após quatro semanas de pós-operatório), com um mínimo de seis meses de seguimento. As medidas de eficácia secundárias, também avaliadas aos seis meses de pós-operatório, foram: i) melhor acuidade visual corrigida (AV), ii) incidência de re-intervenção por HVPO ou revisão da vitrectomia, e iii) qualidade de vida. Os efeitos adversos avaliados foram: i) evolução de catarata, ii) rubeosis iridis ou glaucoma neovascular, iii) descolamento da retina (DR), iv) inflamação intraocular, v) efeitos secundários sistémicos e vi) gravidade da HPVO.

\section{RESULTADOS}

No total, 12 EACs de diferentes países (Irão: 3; Itália e Egipto: 2; Coreia do Sul, EUA, México, Paquistão e Japão: 1) cumpriram os critérios de inclusão da presentes RS, representando 654 olhos de pacientes com a idade média de 54 anos. Nos estudos incluídos, as complicações da RDP que justificaram a cirurgia foram: i) hemorragia vítrea persistente, ii) DR tracional envolvendo a mácula ou iii) DR misto (i.e. regmatogéneo e tracional). Com a excepção de di Lauro 2009, em que o fármaco anti-VEGF foi utilizado três semanas antes da cirurgia, a injeção intravítrea de anti-VEGF foi sempre realizada num período menor que uma semana relativamente à data da cirurgia. $O$ anti-VEGF utilizado foi sempre o bevacizumab, na dose de $1,25 \mathrm{mg} \mathrm{em} 10$ estudos e de 0,16 mg e 2,5 mg em Manabe 2015 e Modarres 2009, respectivamente.

Em todos os estudos foram observadas algumas limitações do ponto de vista metodológico, destacando-se o alto de risco de viés relativo às categorias "Masking participants and investigators (performance bias)" e "Masking of outcome assessment (detection bias)", devido ao desenho open-label de quatro dos EACs. Além disso, a maioria dos estudos não detalhava suficientemente o processo de aleatorização, apresentando, por isso, viés de selecção.

Relativamente à eficácia, os participantes no grupo anti-VEGF apresentaram menor incidência de HVPO às quatro semanas (rácio de riscos (RR) 0,28; $95 \%$ intervalo de confiança $(\mathrm{Cl}) 0,08$ - 0;96; alta qualidade de evidência), comparando com grupo de cirurgia apenas - o que corresponde a menos $13 \%$ de indivíduos com HVPO precoce após vitrectomia por RDP. No entanto, o efeito na HVPO tardia (i.e. aos seis meses) não foi estatisticamente significativo (RR 0,72; $95 \% \mathrm{Cl} 0,30-1,72$; baixa qualidade de evidência), correspondendo a menos $5,5 \%$ dos indiví- duos com HVPO tardia. Os efeitos na AV não foram reportados dada a elevada heterogeneidade entre os estudos. Nenhum dos estudos reportou dados relativos à medição do impacto na qualidade de vida.

Não foram observadas complicações locais ou sistémicas do anti-VEGF, tendo sido o risco de DR inferior nos

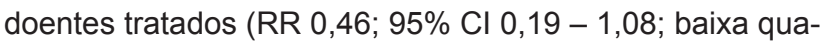
lidade de evidência).

A Tabela 1 apresenta o sumário dos resultados.

\section{CONCLUSÃO}

Os resultados obtidos permitem afirmar que a utilização perioperatória de anti-VEGF diminui a incidência de HVPO precoce após vitrectomia para RDP, sem complicações locais ou sistémicas associadas. No entanto, esse efeito benéfico não se repercutiu na diminuição da HVPO aos seis meses de seguimento.

\section{COMENTÁRIO}

A diabetes mellitus é um problema de saúde pública, constituindo a retinopatia diabética a principal causa de cegueira na população em idade ativa, a nível mundial. ${ }^{2}$ A vitrectomia via pars plana é uma cirurgia necessária e eficaz na resolução de algumas das complicações da RDP. Estima-se também que, das cirurgias de vitrectomia necessárias, $10 \%$ precisem de re-intervenção, sendo a HVPO uma das principais causas. ${ }^{3,4}$ A HVPO (precoce e tardia) tem habitualmente como causas a hemorragia a partir de neovasos remanescentes ou de tecidos manipulados durante a cirurgia. Quando ocorre, atrasa significativamente a recuperação da função visual e limita a capacidade de visualização do fundo ocular. ${ }^{5} \mathrm{~A}$ utilização de fármacos anti-VEGF antes ou no momento da cirurgia tem sido sugerida para a redução de HVPO, contudo sem evidência de boa qualidade que o justificasse. Esta necessidade motivou a presente RS e permitiu gerar alta qualidade de evidência relativamente à utilização pré- ou intraoperatória de anti-VEGF na diminuição da incidência de HVPO precoce (quatro semanas) após vitrectomia para RDP. Tendo em conta que não foram observados efeitos adversos sistémicos ou locais no grupo de tratamento, o efeito benéfico desta intervenção deverá ser tido em conta na abordagem peri-operatória dos doentes com RDP. A ausência de um resultado estatisticamente significativo relativo à ocorrência de HVPO tardia (seis meses) deve servir de incentivo ao desenho e concretização de EACs que permitam esclarecer definitivamente o papel dos anti-VEGF na diminuição da incidência de HVPO.

\section{IMPLICAÇÕES PARA A PRÁTICA CLÍNICA}

- Esta RS demonstrou, com alta qualidade de evidência, que a utilização pré- ou intra-operatória de anti-VEGF diminui a incidência de HVPO precoce (até quatro semanas) após vitrectomia por RDP. No entanto, esse efeito benéfico não se repercutiu na diminuição da HVPO aos 6 meses de seguimento.

- Não foram observadas complicações relacionadas com 


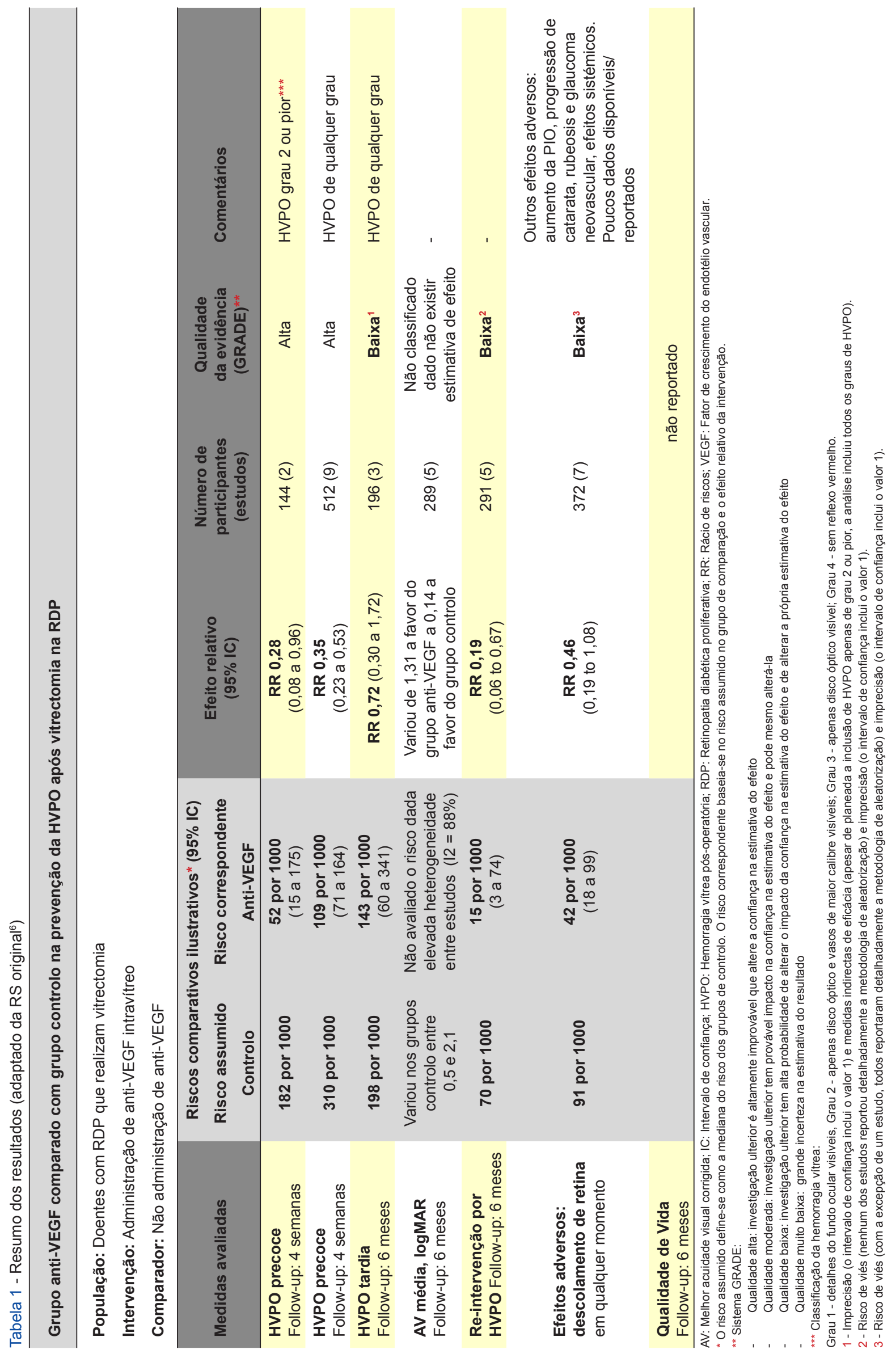


o tratamento anti-VEGF.

- Novos estudos aleatorizados e controlados, com este e outros fármacos anti-VEGF, irão seguramente contribuir

\section{REFERÊNCIAS}

1. Smith JM, Steel DH. Anti-vascular endothelial growth factor for prevention of postoperative vitreous cavity haemorrhage after vitrectomy for proliferative diabetic retinopathy. In: Steel $\mathrm{DH}$, editor. Cochrane Database Syst Rev. Chichester: John Wiley \& Sons; 2011

2. Yau JW, Rogers SL, Kawasaki R, Lamoureux EL, Kowalski JW, Bek T, et al. Global prevalence and major risk factors of diabetic retinopathy. Diabetes Care. 2012;35:556-64.

3. Kaiser RS, Maguire MG, Grunwald JE, Lieb D, Jani B, Brucker AJ, et al. One-year outcomes of panretinal photocoagulation in proliferative diabetic retinopathy. Am J Ophthalmol. 2000;129:178-85.

4. Yorston D, Wickham L, Benson S, Bunce C, Sheard R, Charteris D. para corroborar os achados desta RS e, dessa forma, ajudar os doentes e cirurgiões do seguimento e tratamento cirúrgico da RDP e suas complicações.

Predictive clinical features and outcomes of vitrectomy for proliferative diabetic retinopathy. $\mathrm{Br} \mathrm{J}$ Ophthalmol. 2008;92:365-8.

5. Ferenchak K, Duval R, Cohen JA, MacCumber MW. Intravitreal bevacizumab for postoperative recurrent vitreous hemorrhage after vitrectomy for proliferative diabetic retinopathy. Retina. 2014;34:117781.

6. Smith JM, Steel DH. Anti-vascular endothelial growth factor for prevention of postoperative vitreous cavity haemorrhage after vitrectomy for proliferative diabetic retinopathy. Cochrane Database Syst Rev. 2015;8:CD008214.

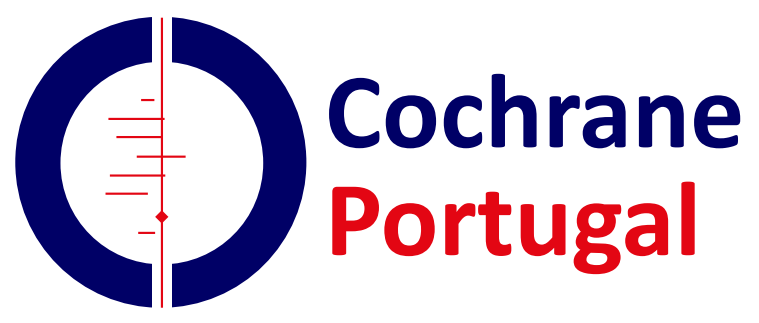

